Cell Reasearch (1993),3,75-83

\title{
Antisense EGFR sequence reverses the growth proper- ties of human liver carcinoma cell line BEL-7404 in vitro
}

\author{
XU Yonghua, WAnli JiAnG, Sufeng PENG, \\ YINGHUA CHEN \\ Laboratory of Cellular and Molecular Oncology, Shanghai \\ Institute of Cell Biology, Chinese Academy of Sciences, \\ Shanghai 200031, China
}

\section{ABSTRACT}

A recombinant plasmid containing a full length human epidermal growth factor receptor (EGFR) cDNA sequence in antisense orientation was transferred into cells of a human liver carcinoma cell line BEL-7404. Compared with the control cell clone JX-0 transferred with the vector plasmid and the parent BEL-7404 cells, the antisense EGFR transferred cell clone JX-1 showed a decreased EGFR gene expression and reduced significantly the growth potential either in anchorage-dependent or anchorage-independent growth. Furthermore, JX-1 cells appeared to be distinctly dependent on serum concentration for monolayer growth. The results suggested that antisense EGFR could partly block the EGFR gene expression and reverse the malignant growth properties of human liver carcinoma cells in vitro.

Key words: antisense EGFR, liver carcinoma cells, cell growth

\section{INTRODUCTION}

Epidermal growth factor (EGF) is a potent mitogen which stimulates the proliferation of many different types of cells [1,2]. The action of EGF is mediated by its own receptor, (EGF receptor, or EGFR) which contains an extracellular binding domain, a transmembrane domain and an intracellular protein kinase domain capable of phosphorylating itself and other proteins on tyrosine residues [3,4]. This phosphorylation is believed to initiate a series of events which eventually result in cell division.

The sequence homology between intracellular portion of the EGFR and gp6 $68^{{ }^{{ }^{-}}{ }_{e r b B} \mathrm{~b}}$, the transforming protein of oncogene v-erbB [5] and the overexpression of EGFR gene 
Antisense EGFR reverses the growth of cancer cells

in many kinds of cancer cells suggested that the EGFR might be responsible for the genesis and the maintenance of malignant properties of certain types of tumor[6-11].

In a previous study we analyzed the EGFR gene expression in BEL-7404 cells. About $1.1 \times 10^{5}$ molecules of EGFR were present on the surface of each BEL-7404 cell which expressed a $170 \mathrm{kD}$ glycoprotein and $5.6 \mathrm{~Kb}$ mRNA of the receptor[12]. We also observed that EGF could stimulate the anchorage-dependent and anchorageindependent growth of BEL-7404 cells in vitro[12]. The addition of antibody against EGFR to culture medium obviously inhibited the growth of BEL-7404 cells (unpublished data). These results suggested that the expression of EGFR would be indispensible for the growth of human liver carcinoma cells. Questions thus raised included whether EGFR expression would be necessary for hepatoma cells and what might happen if EGFR gene expression was blocked in the cells.

To answer these questions, a genetic approach has been carried out by introducing a cloned antisense EGFR sequence into BEL-7404 cells and identifying whether it could specifically eliminate the EGFR gene expression or block its function. We reported here the construction of EGFR antisense sequence and their effects on EGFR synthesis and the growth properties of human liver carcinoma cells. The results indicated that the antisense EGFR sequence was capable of reducing the EGFR gene expression in hepatoma cells and reversing the malignant growth behavior of hepatoma cells in vitro.

\section{MATERIALS AND METHODS}

\section{Cells}

Human liver carcinoma cell line BEL-7404 was established in our Institute. The cells were maintained at $37^{\circ} \mathrm{C}$ in DMEM supplemented with $20 \%$ calf serum, $100 \mathrm{U} / \mathrm{ml}$ of penicillin and 100 $\mu \mathrm{g} / \mathrm{ml}$ of streptomycin in $5 \% \mathrm{CO}_{2}$ incubator.

\section{Plasmid construction}

Full length of EGFR cDNA fragment was prepared by Xba1/Sph1 digestion from pER plasmid DNA. Expressing vector pEV-1 was cleaved by BgIII digestion and ligated with an adaptor which contained a Xba1 and a Sphl sites inside. After insertion, the plasmid vector with Sph1 site at upstream and Xbal site at down-stream was selected. Then the vector was cut with Sph1 and $\mathrm{Xba1}$, and used to ligate with EGF receptor cDNA. The final reconstructed plasmid $\mathrm{pYX}-\mathrm{ER}^{\text {as }}$ contained an EGFR sequence in opposite orientation, with 3' end of the cDNA at up-stream and 5 ' end of the cDNA at down-stream (Fig 1)

\section{DNA transfection}

The recombinant plasmid of antisense EGFR sequence was co-transferred with pSV2-neo into human liver carcinoma cells BEL-7404 by electroporation procedure as described by BIO-RAD company. The parent vector plasmid pEV-1 was used as control for transfection. After electroporation, cell suspension was plated into dishes and maintained at $37^{\circ} \mathrm{C}$ for $24 \mathrm{~h}$. On the following day, the medium was replaced by DMEM without DNA; and after another $48 \mathrm{~h}$, the cells were placed under G418(400 $\mu \mathrm{g} / \mathrm{ml})$ selection. Single colonies appeared $14 \mathrm{~d}$ later and were isolated by trypsin treatment and cloning. 


\section{Growth assay}

Cell growth was monitored by ${ }^{3} \mathrm{H}$-thymidine incorporation and cell number counting of the culture in the presence of $20 \%$ calf serum. The detection of anchorage-independent growth of the cells in soft-agar was performed as described elsewhere [12]. The comparison of serum dependence of cell growth was performed by routine culture procedure in the medium containing different concentrations of serum.

\section{Immunoblotting}

Cells lysates were separated on SDS-PAGE and blotted onto nitrocellulose filter (S\&S BA85, $0.45 \mu \mathrm{m})$ as described previously[12]. Antibody against human EGFR protein was used as probe to explore the expression of EGFR in cells by Vectastain ABC Kit according to the protocol recommended by Vector Laboratories, Inc.

\section{RESULTS}

Selection and general morphological characterization of antisence EGFR transfectants

Stable antisense EGFR transfectants and control transfectants transferred with vector containing no exogenous insert DNA were obtained by co-transfecting with pYX-ER ${ }^{a s}$ plus pSV2- neo or pEV-1 plus pSV 2-neo respectively and selected in the presence of G418 ( $400 \mu \mathrm{g} / \mathrm{ml})$, under which all the parent liver carcinoma cells BEL7404 died after a $14 \mathrm{~d}$ treatment. JX-1, one of the single colonies transfected with antisense EGFR and JX-0, one of the single colonies transfected with vector alone were maintained in $20 \%$ bovine calf serum containing medium at $5 \% \mathrm{CO}_{2}$ incubator.

A general morphological characterization was compared between JX-1 cells, JX0 cells and parent BEL-7404 cells. As shown in Fig 2, the JX-1 cells exhibited a growth morphology different from that of BEL-7404 and JX-0 cells, although they were inoculated at the same initial concentration and cultured under the same condition. JX-1 cells (Fig 2,A) seemed to grow much slowly, appeared round or spindle in shape, smaller in size and not confluent. On the other hand, the BEL-7404 cells and JX-0 cells (Fig 2,B and C) could grow into a big crowd at a high density and with many overlaps among the cells. Most of the BEL-7404 cells and also JX-0 cells appeared larger in size than that of JX-1 cells.

\section{EGFR expression in antisense EGFR transfectants}

EGFR expression in antisense EGFR transfected JX-1 cells was compared with the parent hepatoma cells BEL-7404 and the vector plasmid transfected JX-0 cells by immunoblotting detection, using polyclonal anti-human EGFR antibody as probe. It should be mentioned that the protein contents of Cell lysates of 3 different cell lines used in SDS-PAGE was strictly matched. Fig 3 showed that the expression of EGFR in JX-1 cells was obviously lowered. An inhibition of about 50\% was seen in JX-1 cells as compared with BEL-7404 cells, but no significant reduction of EGFR expression could be found in JX-0 cells. The results suggested that the antisense EGFR sequence might efficiently interfere with the EGFR gene expression 
Antisense EGFR reverses the growth of cancer cells

in hepatoma BEL-7404 cells.

Anchorage-dependent and anchorage-independent growth of antisense EGFR transforments

The anchorage-dependent growth of JX-1 cells, as shown in Fig 4, was much
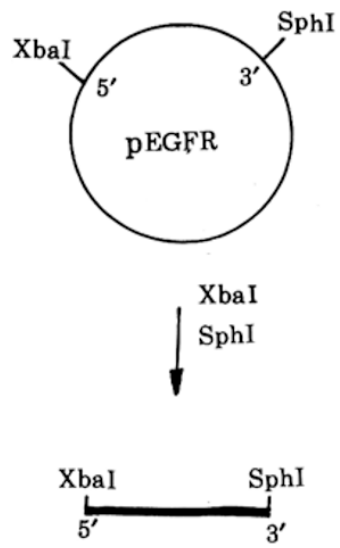

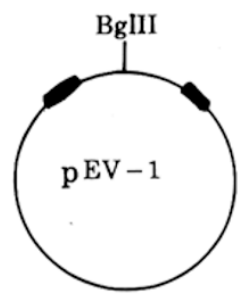

$\uparrow^{\text {BgIII }}$

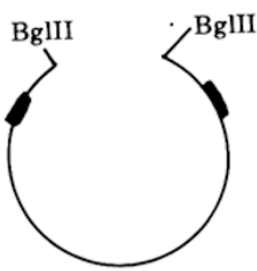

1

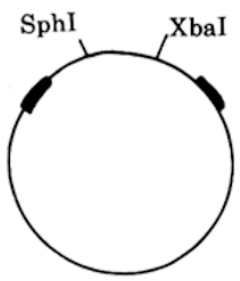

SphI

XbaI
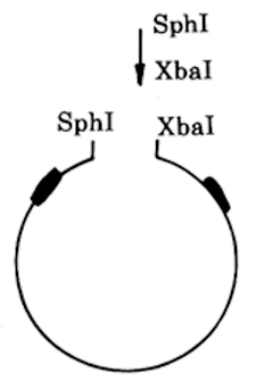

Adaptor

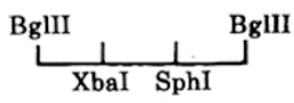

Ligation

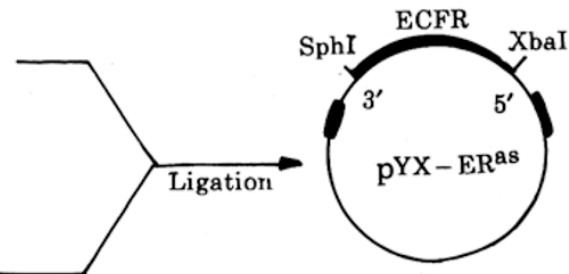

Fig 1. Construction of $\mathrm{pYX}-\mathrm{ER}^{\mathrm{as}}$. 
$\mathrm{Xu}$ YH et al.

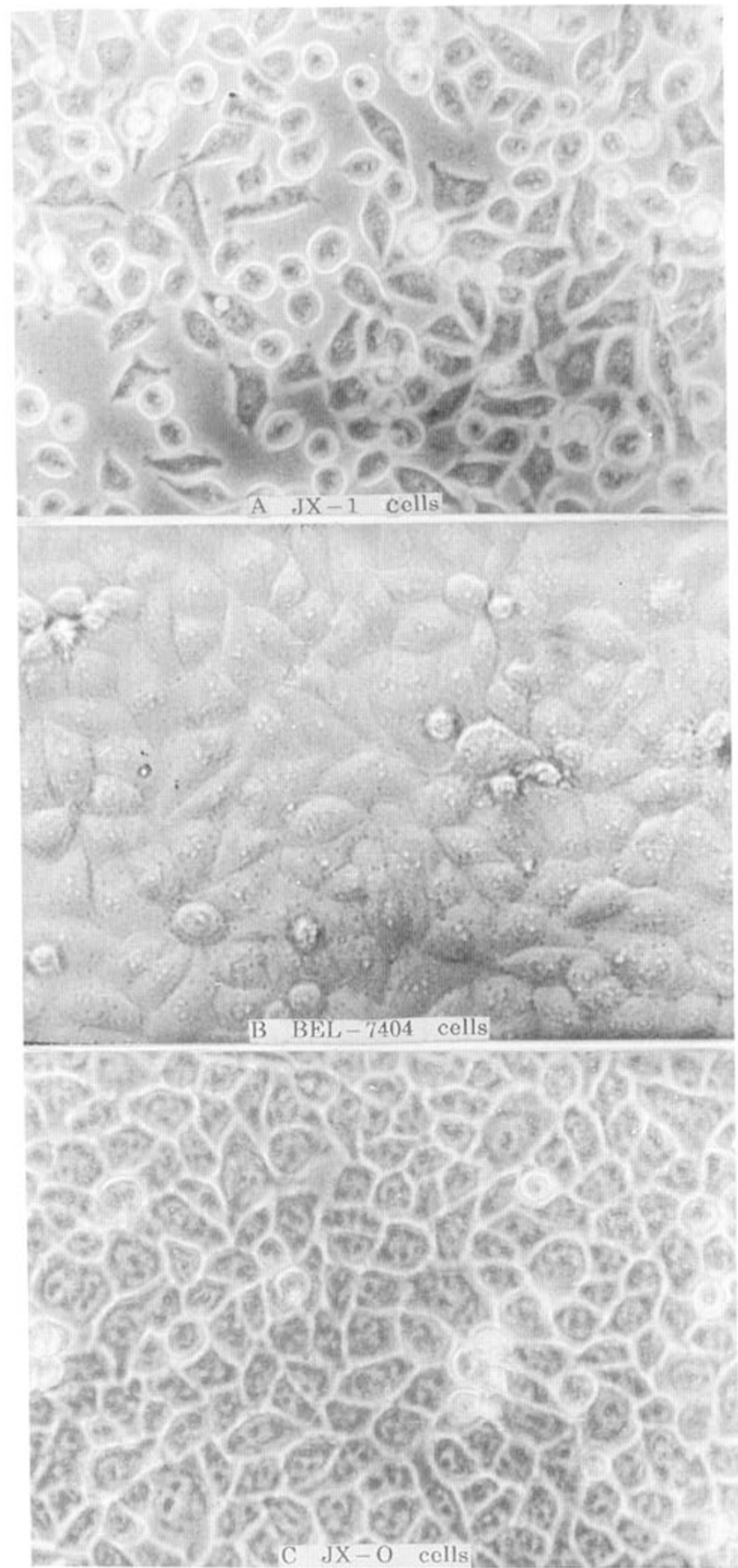

A. JX-1 cells

B. BEL-7404 cells

C. .JX-0 cells

Fig 2. Morphological characteristics of JX-1 cells (A), BEL-7404 cells (B) and JX-O cells $($ C $) \times 400$. 
Antisense EGFR reverses the growth of cancer cells

BEL-

7404
$J X-1 \quad J X-0$

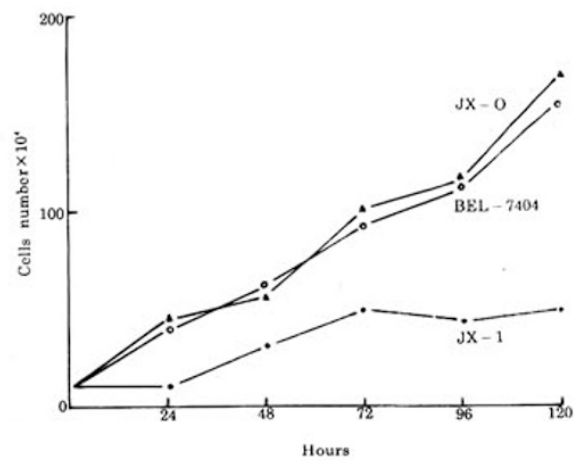

Fig 4. $\triangle$ Anchorage dependent growth of JX-1, JX- 0 and BEL-7404 cells in $10 \%$ bovine serum containing medium

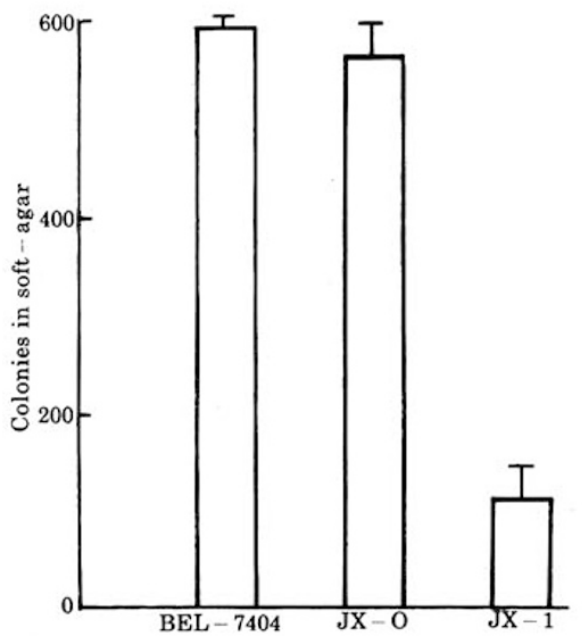

Fig 5. $\triangle$ Colony formation of JX-1, JX-0 and BEL-7404 cells in softagar hibition of confluent JX-1 cells in monolayer culture.

The potentiality of anchorage independent, growth of JX-1, JX-0 cells and parent BEL-7404 cells was shown in Fig 5. The results indicated that JX-1 cells could only produce much fewer colonies in soft agar than BEL-7404 cells did, yet no detectable 
reduction was observed in case of $\mathrm{J} \mathrm{X}-0$ cells. The number of colonies of $\mathrm{JX}-1$ cells in soft agar after a 2 weeks culturing was only one sixth to one fifth of that of JX-0 and BEL-7404 cells.

\section{Serum dependence of growth of antisense EGFR transfectants in culture}

Medium containing various amount of serum were prepared and served to analyze the serum dependence of growth for JX-1 cells. As shown in Tab 1, BEL-7404 cells grew vigorously in either $10 \%$ serum medium or low serum medium, even in serum free medium. A very similar growth potentiality in low serum medifim and a slight reduction of growth rate in serum-free medium was found with JX-0 cells. In contrast, the growth of JX-1 cells was found to be highly serum dependent. In 10\% serum containing medium, the antisense EGFR transforments grew much slower than their parent cells ( Tab 1 and Fig 4 ). Medium containing less than 10\% serum did not support their growth and proliferation. In medium with $5 \%$ or less serum, most of JX-1 cells no longer grew and some cells died within 5 days.

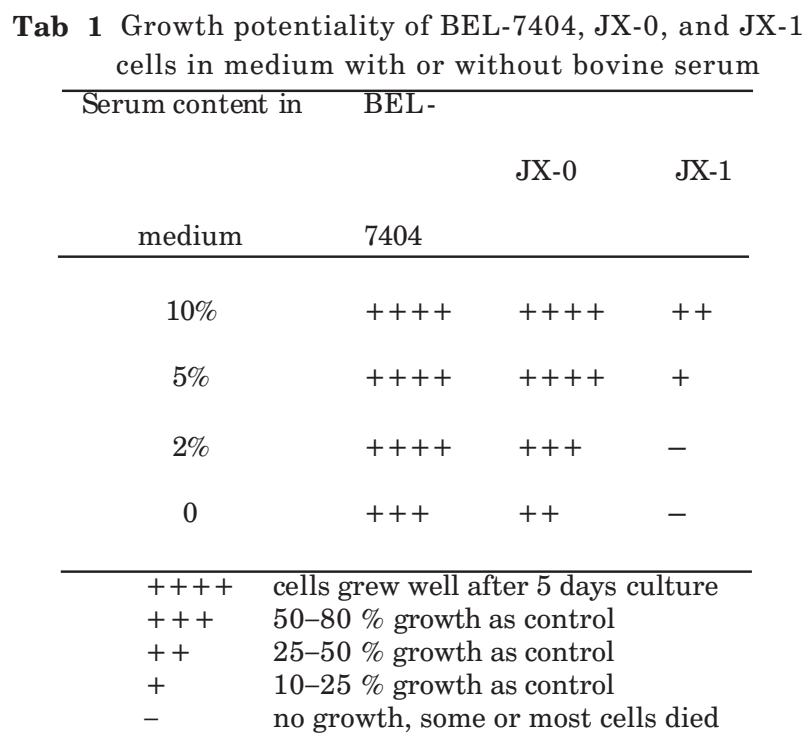

\section{DISCUSSION}

Many lines of evidence have been reported to suggest that the EGFR was related to cell transformation. A high level of homology between the amino acid sequence of the human EGFR and the avian V-erbB oncogene product[5] and the overexpression of EGFR in a number of human cancer cell lines and tumor tissues[6-9,13] indicated an important role of EGFR in cell transformation and tumorigenesis. It was also found that the EGFR gene transfected NIH3T3 cells displayed a high level of EGFR, formed colonies in soft agar in the presence of EGF and produced tumor 
Antisense EGFR reverses the growth of cancer cells

in nude mice [14]. EGFR was overexpressed in some human liver carcinoma cells lines[12], but the actual function of EGFR in liver carcinoma cells remained unclear. BEL-7404 cells contained an elevated level of EGFR at $1.1 \times 10^{5}$ molecules/cell [12]. Addition of exogenous EGF stimulated both anchorage-dependent and anchorageindependent growth of BEL-7404 cells. Antibody against EGFR added to culture medium inhibited the growth of the cells. These results implied that EGFR might piny a modulatory role in the proliferation of hepatoma cells.

In this paper, we reported that the transfection with vector containing full length antisence EGFR sequence might regulate the EGFR gene expression in the recipient cells, resulting in reduction of $170 \mathrm{kD}$ EGFR protein to about $50 \%$ of the amount present in parent cells. A similar effect of antisense sequence of 5'-end of EGFR cDNA was identified when the vector was introduced into human sequmous carcinoma cell line yielding a decrease of up to 52\% of EGFR synthesis[15]. Interestingly, we found that the reduction EGFR expression in EGFR antisense transfectants was accompanied by a significant inhibition of cell proliferation and soft agar colony formation, and also the dramatic dependence of serum concentration for cell growth in culture medium as well. The transformed cells or malignant cells usually showed an altered cellular morphology and growth characteristics, e.g. the different shape and appearance from its normal counterpart, the increased saturation density and decreased serum factors requirements, the loss of dependence on anchorage for growth and contact inhibition of movement, the secretion of transforming growth factors etc. As compared with BEL-7404 human liver carcinoma cells which possessed typical malignant growth characteristics as shown in this report, antisense EGFR transfected hepatoma cells, JX-1 cells, have been deprived their major malignant growth properties. The data presented here seemed to indicate a correlation between the level of EGFR expression and the extent of the reversion of malignant phenotype of human liver carcinoma cells in vitro. It was also true that EGFR level on A431 cell surface was closely related to its tumorigenetic potential in animals.

Carcinogenesis may be defined as a series of progressive changes of genetic events in the cells. The liver carcinoma may be evolved as a result of the accumulation of multistep genetic changes of the protooncogenes and other related genes involved in the signal transduction pathway. Modulations of these genes could be one of the approaches to suppress the malignant behavior of the cancer cells. The intriguing antisense technique and its potential application has been an interesting subject in the gene therapy for cancer. The mechanism by which antisense sequence functions remains to be explored. The inhibition of some harmful genes such as oncogenes and genes that are required for the autocrine growth of cancer cells by antisense sequences may be somehow involved in the molecular events of their action.

\section{REFERENCES}

[1] Cohen S. The epidermal growth factor. Cancer 1983; 51:1787-91 
[2] Carpenter G, Cohen S. Human epidermal growth factor and the proliferation of human fibroblasts. J Cell Physiol 1975; 88:227-38.

[3] Carpenter G. Receptors for epidermal growth factor and other polypeptide mitogens. Ann Rev Biochem 1987; 56:881-94.

[4] Downward J, Pareker P, Waterfield M. Autophosphorylation site on the epidermal growth factor receptor. Nature 1984; 311:483-5.

[5] Downward J, Yarden Y, Mayes E, Scrace G, Totty N, Stockwell P, et al. Close similarity of epidermal growth factor receptor and v-erbB oncogene protein sequences. Nature 1984; 307:5217.

[6] Xu YH, Richert N, Ito S, Merlino G, Pastan I. Characterization of epidermal growth factor receptor gene expression in malignant and normal human cell lines. Proc Natl Acad Sci USA 1984; 81:7308-12.

[7] Libermann T, Nusbaum H, Razon N, Kris R, Lax I, Soreq H. et al. Amplification, enhanced expression and possible rearrangement of EGF receptor gene in primary human brain tumors of glial origin. Nature 1985; 313:144-7.

[8] Xu YH, Ishii S, Clark A, Sullivan M, Wilson R, Ma D, et al. Human epidermal growth factor receptor cDNA is homologous to a variety of RNAs overproduced in A431 carcinoma cells. Nature 1984; 309:806-10.

[9] Ullrich A, Coussens L, Hayflick J, et al. Human epidermal growth factor receptor cDNA sequence and aberrant expression of the amplified gene in A431 epidermoid carcinoma cells. Nature 1984; 309:418-24.

[10] Lin C, Chen W, Kruiger L, Stolarsky S, Weber W, Evans R. et al. Expression and cloning of human EGF receptor complimentary DNA: gene amplification and three related messenger RNA products in A431 cells. Science 1984; 224:843-8.

[11] Filmus J, Pollak M, Caillean R, Buick R. MDA-468, a human breast cancer cell line with a high number of epidermal growth factor(EGF) receptors, has an amplified EGF receptor gene and is growth inhibited by EGF. Biochem Biophys Res Comm 1985; 128:898.

[12] Xu YH, Jiang WL, Peng SF. EGFR expression and EGF stimulation of proliferation in human liver carcinoma cells. Acta Biol Exp Sin 1989; 22:445-53.

[13] Ozanne B, Richards S, Hendler F, Burns D, Gusterson B. Overexpression of the ECF receptor is a hallmark of sequamous cell carcinomas. J Pathol 1986; 149:9-14.

[14] Veru T, Beguinot L, Vass W, Willingham M, Merlino C, Lowy D. Epidermal growth factor dependent transformation by a human EGF receptor proto-oncogene. Science 1987; 213:140810 .

[15] Hunts J, Merlino G, Pastan I, Shimizu N. Reduction of EGF receptor synthesis by antisense RNA vectors. FEBS Letters 1986; 206:319-22.

Received 8-7-1992. Revised 21-8-1992. Accepted 25-8-1992 